\title{
Mechanisms for Reducing Low Back Pain: A mediation analysis of a multifaceted intervention in workers in elderly care
}

\author{
Matthew L Stevens, ${ }^{1}$ Eleanor Boyle, ${ }^{2}$ Jan Hartvigsen,,${ }^{2,3}$ Gemma Mansell, ${ }^{4}$ Karen Søgaard,${ }^{2}$ Marie \\ B Jørgensen, ${ }^{5}$ Andreas Holtermann, ${ }^{2,5}$ Charlotte D N Rasmussen ${ }^{5}$ \\ ${ }^{1}$ Musculoskeletal Health Sydney, School of Public Health, The University of Sydney, Sydney, \\ Australia. \\ ${ }^{2}$ Department of Sports Science and Clinical Biomechanics, University of Southern Denmark, \\ Odense, Denmark \\ ${ }^{3}$ Nordic Institute of Chiropractic and Clinical Biomechanics, Odense, Denmark \\ ${ }^{4}$ Research Institute for Primary Care \& Health Sciences, Keele University, Staffordshire, UK \\ ${ }^{5}$ The National Research Centre for the Working Environment, Copenhagen, Denmark
}

\section{Correspondence to: Matthew L Stevens}

E-mail address: matthew.stevens@sydney.edu.au

Contact address: Musculoskeletal Health Sydney, School of Public

Health, The University of Sydney, PO Box M179, Missenden Rd, NSW, 2050, Australia, T: +61286276268

Keywords: workplace interventions; cognitive behavioural training; physical training; participatory ergonomics; musculoskeletal disorders; nurses' aides;

Manuscript size: 3740 words (24,957 characters with spaces); 6 figures, 1 table

\section{What is new in the paper?}

This was the first study to use mediation analyses to investigate mechanisms of a multifaceted workplace intervention for reducing low back pain in workers in elderly care. The study found that decreasing fear-avoidance and increasing the use of assistive devices do not seem to be ideal targets for reducing low back pain in this population. 


\section{Abstract}

\section{Purpose}

A multifaceted workplace intervention consisting of participatory ergonomics, physical training, and cognitive-behavioural training (CBT) has shown effectiveness for reducing low back pain (LBP). However, the mechanisms of action underlying these intervention components are not well understood.

\section{Methods}

This was a mediation analysis of a cluster-randomised controlled trial of a multifaceted intervention in 420 workers in elderly care. Mediation analysis was carried out via structural equation modelling. Potential mediators investigated were: fear avoidance beliefs, perceived muscle strength, use of assistive devices at work and perceived physical exertion at work. LBP outcomes assessed were: days with LBP, LBP intensity and days with bothersome LBP.

\section{Results}

There were no significant indirect effects of the intervention on LBP outcomes. There were significant effects of the intervention on both fear avoidance measures $(\beta=-0.63,95 \% \mathrm{CI}[0.03$, $1.23] ; \beta=-1.03,95 \% \mathrm{CI}[0.34,1.70])$ and the use of assistive devices $(\beta=-0.55,95 \% \mathrm{CI}[0.05$, $1.04])$, but not on perceived muscle strength $(\beta=-0.18,95 \% \mathrm{CI}[-0.13,0.50])$ or physical exertion $(\beta=-0.05,95 \% \mathrm{CI}[-0.31,0.40])$. The only potential mediator with a significant effect on LBP outcomes was physical exertion, which had a significant effect on LBP intensity $(\beta=0.14$, $95 \% \mathrm{CI}[0.04,0.23])$.

\section{Conclusions}

Page 2 of 30 
A multifaceted intervention consisting of participatory ergonomics, physical training, and CBT was able to decrease fear avoidance beliefs and increase use of assistive devices in the workplace. However, these changes did not explain the effect of any of the intervention components on days with LBP, LBP intensity and days with bothersome LBP.

Page 3 of 30 


\section{Introduction}

Globally, low back pain (LBP) is very common and its consequences are a major burden on society. With a mean global point prevalence of around 18\% (Hoy et al. 2012), it is the leading cause of years lived with disability (Vos et al. 2012) and ranks third when measured in disabilityadjusted life years (Murray and Lopez 2013). Those with LBP are less able to work and it is the leading chronic health condition that forces older workers out of the workforce (Schofield et al. 2008). This results in severely decreased wealth for individuals (Schofield et al. 2011, 2015) and lost productivity for industry (Mannion et al. 2009; Wieser et al. 2011; d'Errico et al. 2013). Due to the severe impact of LBP on both individuals and industry, workplace interventions aimed at reducing LBP are becoming increasingly common.

There are several interventions that have shown a beneficial effect on LBP in the workplace. Such interventions include participatory ergonomics (Rivilis et al. 2008; Driessen et al. 2010), physical training (Tveito et al. 2004; Driessen et al. 2010) and cognitive-behavioural training (CBT) (van Tulder et al. 2001; Tveito et al. 2004; Kamper et al. 2015). However, although these interventions show benefit, the mechanisms behind their effect are not well understood. Current theories suggest these interventions function by changing workers' attitudes and beliefs with respect to LBP and performance of their work tasks (Laisné et al. 2012) (e.g. CBT changing fear-avoidance beliefs) or by changing the physical relationship between individuals and their work tasks (Sjøgaard et al. 2014) (e.g. by making work easier through physical training to increase an individual's physical capacity or by decreasing workload through participatory ergonomics).

To test these hypothesised mechanisms, a statistical test (called mediation analysis) is used. Mediation analyses quantify causal mechanisms by splitting the causal effect of the intervention on the outcome into the indirect effect (the effect which acts through the proposed mediator) and

Page 4 of 30 
direct effect (all other unspecified mechanisms) (Mansell et al. 2013; Lee et al. 2017). Despite mediation analysis being the standard, few studies have used this method to investigate mechanisms of effect in LBP (Mansell et al. 2013). To date, such analyses are limited to clinical

populations, such as patients with chronic LBP (Spinhoven et al. 2004; Smeets et al. 2006; Hall et al. 2016; Mansell et al. 2016a), with no studies having yet investigated mediating mechanisms of LBP interventions in workplace settings. They have also focused on understanding the relationship between pain and disability (Lee et al. 2015, 2016) or investigations of potential psychological mediators, such as catastrophising (Lee et al. 2016) or fear avoidance (Mansell et al. 2016a; Whittle et al. 2017), rather than physical factors such as muscle strength.

To fill these evidence gaps and investigate whether the proposed interventions worked through the mechanisms as hypothesised, we performed a mediation analysis of a multifaceted intervention conducted in the workplace. The intervention included participatory ergonomics, physical training, and cognitive-behavioural training (CBT), and has already shown to be effective at reducing days with LBP, reducing LBP intensity and reducing the number of days with bothersome LBP (Rasmussen et al. 2015). We sought to determine whether changes in fear avoidance beliefs, perceived muscle strength, use of assistive devices at work or perceived exertion at work mediated this effect. Our hypothesis was that the intervention would cause significant change in our proposed mediators which would in turn cause significant change in the LBP outcomes measured.

\section{Methods}

This study is a secondary mediation analysis of a pragmatic, step-wedged, cluster-randomized controlled trial conducted in Denmark from November 2012 to May 2014. Approval for the study

Page 5 of 30 
was provided by the Danish Data Protection Agency and the Ethics Committee for the regional capital of Denmark (Journal number: H-4-2012-115). The trial was registered with the Current Controlled Trials Register (ISRCTN78113519). Full details of the methods used are provided in the previously published protocol (Rasmussen et al. 2013) and primary paper (Rasmussen et al. 2015), but are briefly described below.

\section{Participant Recruitment}

Participants were recruited through public elderly-care service providers within a large municipality of Denmark. Nine districts within the municipality were offered participation, of which four accepted. All employees within these four districts were then sent a short information brochure describing the aim and activities of the study and invited to a 30-minute meeting in which employees could express their interest in being involved. Included participants were workers in elderly care (nurses' aides and service workers (e.g. janitors, kitchen and cleaning personnel)) employed in elderly care either in nursing homes or in home-care for more than 20 hours per week and aged 18 to 65 years. Participants were excluded if they were unwilling to participate, on long-term sickness absence or not permanently employed.

\section{Randomisation and blinding}

A balanced cluster randomisation was applied to allocate each of the clusters to one of four successive time periods, three months apart as per the stepped wedge design (Figure 1). A cluster consisted of a working team that comprised of a supervisor and the workers who reported to that supervisor. Although blinding of participants was not possible due to the nature of the trial, concealed allocation was applied. Moreover, the participants did not receive information about which group they were randomised to until shortly before crossing over from control to 
intervention. Follow-up data collection was performed using standardised text messages. All persons collecting/handling data were blinded to group allocation.

\section{Intervention}

The intervention lasted for three months and was conducted on-site during the participants' working time. It consisted of three primary components: participatory ergonomics, physical training and CBT (Figure 2). All components were delivered by a therapist (physiotherapist or occupational therapist). Each therapist received six days of training in the study intervention and received written materials reinforcing the training. They were also provided with ongoing support throughout the study. All sessions were conducted in a group setting based on working teams.

Figure 1. Overview of the stepped-wedge design used for the randomised-controlled trial of a multifaceted intervention in workers in elderly care.

Figure 2. Timeline for each component of the multifaceted intervention for reducing low back pain in workers in elderly care.

\section{Participatory ergonomics programme}

The participatory ergonomics training was conducted in groups of 5-7 participants and consisted of two 3-hour workshops and two 1-hour evaluation sessions. The workshops were conducted during weeks two and four of the intervention and focused on reducing physical exertion at work by modifying the work tasks perceived as physically demanding and preventing pain by minimising exposure to risk factors for LBP as they were perceived by the participants. The two 1-hour evaluation sessions were run during weeks eight and 12. During these sessions, the participants, with support from the therapist, assessed how well each working team had implemented the ideas provided during the workshops and made suggestions for improvement (Rasmussen et al. 2017). 


\section{Cognitive behavioural training programme}

Two therapist-led 3-hour CBT workshops were conducted during weeks three and five. These workshops focused on the use of cognitive processes to modify maladaptive pain behaviours and thus prevent/reduce pain and the negative effects of pain. The programme was modified from previous programmes (Linton et al. 2005; Jørgensen et al. 2011) and provided an understanding of pain and the pain experience, how physical activity may negatively or positively relate to pain and the ability to function despite pain. It also provided training in specific skills such as problem-solving and pain coping.

\section{Physical training programme}

The physical training consisted of 12 weekly 1-hour sessions during which participants were introduced to, and conducted, various types of physical activity. These were body awareness/postural training, strength and coordination training, or general physical activity. The therapists initially prescribed the training to be conducted. However, from week five onwards participants chose their preferred type of physical activity. The therapists supervised all activities conducted during all 12 sessions.

\section{Outcomes}

Our outcomes of interest were clinical outcomes for LBP. Three measures were chosen: 1) the number of days with LBP in the preceding month - "During the previous four weeks, how many days have you had low back pain?" (0-31 days); 2) LBP intensity - the worst LBP experienced in the preceding month on a 0 to 10 numeric scale - "What was the highest intensity of your low back pain, with 0 being no pain and 10 being the worst imaginable pain?" (0-10 pain); and 3$)$ the number of days with bothersome LBP in the preceding month - "How many days during the 
Mechanisms for Reducing Low Back Pain

previous four weeks has your low back pain been bothersome (i.e. affected your daily activities or routines)?" (0-31 days). These outcomes were chosen based on results of a Delphi study involving international LBP experts that reached consensus about duration and severity of LBP as a minimal definition of LBP (Dionne et al. 2008). We also collected demographic (sex, age, ethnicity), work-related (job group, education, job seniority, job type, work shift, education), and health (smoking, LBP) information.

\section{Potential Mediators}

We investigated four different potential mediators. The hypothesised pathways of the action for the effect of the intervention on LBP outcomes, incorporating the potential mediators, is presented in Figure 3.

- Fear avoidance was measured using two items from the Örebro Musculoskeletal Pain Screening Questionnaire (Linton et al. 2010). These were 1) "If my pain gets worse it is a sign that I should stop what I am doing until the pain decreases" and 2) "I should not do my normal activities or my normal work with the pain I have now." Both these items were rated on a scale from 0-10 with 0 indicating strongly disagree and 10 indicating strongly agree.

- Muscle strength was measured using the question "How would you score your muscle strength compared to people of your own age and sex?" rated on a 0-10 numeric rating scale with 0 indicating much weaker and 10 indicating much stronger compared to others of the same age and sex (Strøyer et al. 2007).

- Use of assistive devices at work was measured by asking "How many times have you lifted or moved things or people without using the necessary assistive devices within the last week?" Responses could range from 0-99.

Page 9 of 30 
- $\quad$ Physical exertion at work was measured using a modified Borg CR10 scale asking the question "How would you rate your physical exertion during your current work?" With a score of 0 indicating not strenuous and 10 indicating maximal strain (Borg 1962; Borg and Kaijser 2006).

\section{Figure 3. Hypothesised pathways of action for the effect of the multifaceted intervention on} low back pain outcomes.

\section{Data collection and follow-up}

Baseline information was collected in-person at the worksite. Participants completed an electronic questionnaire containing demographic information and baseline responses for the outcomes and potential mediators to be investigated. All follow-up data was collected by text message. LBP outcomes were collected every month. Potential mediators were collected every three months. For those not responding to the initial text, a reminder was sent two days later. Those who still did not respond were called by the research team and asked for their response over the phone.

\section{Statistical analysis}

To investigate the indirect effect of the potential mediators on the outcomes of interest, three multi-level multi-mediation structural equation models (SEM) were developed (one for each LBP outcome). Beta-coefficients were derived from the models and represent the mean change (point difference) in the considered mediator/outcome. Each model developed incorporated all potential mediators, assessing the indirect effects of the potential mediators together. Models developed are shown in Figures 4-6. Multi-level modelling accounted for the effect of intra-participant and intra-group correlation. Analyses were conducted using the mean-and-variance adaptive GaussHermite quadrature method (Rabe-Hesketh et al. 2005), the standard method for conducting SEM in STATA. To speed up the iteration process and facilitate convergence the model could be

Page 10 of 30 
approximated using other iterative methods (i.e. a non-adaptive Gauss-Hermite quadrature or mode-and-curvature adaptive Gauss-Hermite quadrature) as needed. The mean-and-variance adaptive Gauss-Hermite quadrature method would then be run to obtain the result. All analyses were conducted using STATA v13. It was hypothesised that allocation to the intervention would significantly reduce fear avoidance beliefs and physical exertion at work; and significantly increase muscle strength and use of assistive devices. It was then hypothesised that these changes in the potential mediators would lead to subsequent significant decreases in the number of days with LBP, LBP intensity and days with bothersome LBP.

\section{Time points utilised}

Due to the stepped-wedge design of the trial, in which participants serve as their own controls, selection of time points that met the needs of the mediation analysis was complex. Analyses were conducted as per the three-month timescale of the intervention. For the intervention group, change in potential mediators were measured over the intervention period (three-months) with change in LBP outcomes measured over the following month. Thus, a participant could only contribute one intervention period. For example, (using Figure 1) for a participant in group 1 the potential mediators were measured as the change in each mediator from time point 3 to time point 6 and the change in the outcomes were measured as the change in each outcome from time point 6 to time point 7 . Time points used for the control group were the same as for the intervention; however, for the control group, all eligible three-month blocks in which the participant was in a control period were used. This meant that a single participant could contribute more than one control period. Control periods immediately prior to the intervention (e.g. Figure 1: Group $2-$ time points 3 to 6) were removed from the analyses as their measurement of LBP outcomes took place within the intervention period (e.g. Figure 1: Group 2 - time points 6 to 7).

Page 11 of 30 


\section{Sensitivity Analyses}

We conducted sensitivity analyses to assess if the presence or absence of pain at baseline or adherence to each component of the intervention influenced any indirect effect of the assessed mediators. Presence or absence of pain was dichotomised on whether there was any reported pain for that outcome at baseline (i.e. reporting at least one day with pain, 1/10 maximal pain intensity or one day of bothersome LBP). Satisfactory adherence to each component of the intervention was defined as a minimum attendance of $50 \%$ for the sessions of that component. All sensitivity analyses were conducted as per the primary analyses. It was hypothesised that adherence with each intervention component would increase its effect on the relevant potential mediator/s. Thus, adherence to the CBT programme was expected to increase the effect of the intervention on fear avoidance, adherence to the PT programme to increase the effect on muscle strength, and adherence to the PE programme to increase its effect on use of assistive devices and physical exertion at work.

\section{Results}

Fifty-four teams containing 1074 workers were identified and considered eligible for the trial. Of these, 21 clusters containing 594 participants were enrolled into the trial and randomised to one of the intervention groups. Of the 594 participants enrolled, 174 participants were missing data relevant to this study ( 8 through technical error, 166 through non-responsiveness) and were thus removed from the analyses. This left 420 participants who provided data for this analysis. Among those not contributing to the analysis, there was a higher rate of smoking and lower job seniority.

\section{Participants}

Participants contributing to these analyses were predominately female (93.5\%), nurses' aides (89.6\%) and working day shift $(80.3 \%)$. The majority $(85.4 \%)$ had been performing their job for

Page 12 of 30 
more than one-year. Although the mean body mass index (BMI) score was 34.4, 88.2\% of participants considered themselves of fair health or better. Just over $60 \%$ of participants had at least one day with LBP in the previous four weeks. Full details are presented in Table 1.

Table 1. Demographics and baseline characteristics for workers in elderly care participating in the mediation analyses

\section{Primary Analyses}

There were no significant indirect effects identified of the intervention on LBP outcomes. There were significant effects of the intervention on decreasing fear avoidance $(\beta=-0.63,95 \% \mathrm{CI}[0.03$, $1.23] ; \beta=-1.03,95 \% \mathrm{CI}[0.35,1.70])$ and increasing the use of assistive devices $(\beta=-0.55$, $95 \% \mathrm{CI}[0.05,1.04])$, but not on perceived muscle strength $(\beta=-0.18,95 \% \mathrm{CI}[-1.32,0.50])$ or physical exertion $(\beta=-0.05,95 \% \mathrm{CI}[-0.31,0.40])$. The only potential mediator with a significant effect on LBP outcomes was physical exertion, which had a significant effect on LBP intensity ( $\beta$ $=0.14,95 \% \mathrm{CI}[0.04,0.23])$. Full details are presented in Figures 4-6.

Figure 4. Effect of the potential mediators on the number of days with low back pain

Figure 5. Effect of the potential mediators on low back pain intensity

Figure 6. Effect of the potential mediators on the number of days with bothersome low back pain

\section{Sensitivity Analyses}

Only one of the sensitivity analyses showed a significant indirect effect. In those with greater than $50 \%$ adherence to the participatory ergonomics programme, one of our fear-avoidance measures had a significant indirect effect of the intervention on the number of days with bothersome LBP.

Page 13 of 30 
However, this relationship, which occurred in our first fear-avoidance measure, was opposite to that hypothesised: although the intervention significantly lowered fear avoidance beliefs ( $\beta$ $=-0.72 ; 95 \% \mathrm{CI}[0.07,1.36])$ this led to an increase in the number of days with bothersome $\operatorname{LBP}(\beta$ $=-0.14 ; 95 \% \mathrm{CI}[-0.27,-0.01])$.

In those with no pain at baseline, there were significant effects of physical exertion on the number of days with LBP $(\beta=-0.07 ; 95 \% \mathrm{CI}[-0.13,-0.01])$ and muscle strength on LBP intensity $(\beta=$ $0.13 ; 95 \% \mathrm{CI}[0.01,0.25])$. However, these effects were opposite to what was hypothesised (i.e. increased physical exertion decreased the number of days with LBP and increased muscle strength increased LBP intensity). In those with pain at baseline increased muscle strength led to decreases in the number of days with $\operatorname{LBP}(\beta=-0.55 ; 95 \% \mathrm{CI}[-0.91,-0.19])$ and pain intensity $(\beta$ $=-0.16 ; 95 \% \mathrm{CI}[-0.31,-0.00])$, but not in bothersome LBP $(\beta=0.08 ; 95 \% \mathrm{CI}[-0.39,0.56]) . \mathrm{In}$ those with pain at baseline, there was also a slight increase in the effect of physical exertion on LBP intensity $(\beta=0.19 ; 95 \% \mathrm{CI}[0.05,0.33])$.

Adherence to the cognitive behavioural therapy programme did trend towards an increased effect on fear avoidance beliefs; however, there was no significant effect on LBP outcomes. Full details are presented in Appendices 1-15.

\section{Discussion}

\section{Summary of findings}

None of the proposed mediators (i.e. fear avoidance, muscle strength, use of assistive devices, perceived physical exertion) showed a significant indirect effect of our intervention on LBP outcomes (i.e. days with LBP, LBP intensity and days with bothersome LBP). The intervention did significantly reduce fear-avoidance beliefs and increase use of assistive devices at work, but

Page 14 of 30 
these did not translate to changes in any of the LBP outcomes. We also found that reducing perceived physical exertion at work significantly reduced LBP intensity.

\section{Strengths and limitations of the study}

The primary strengths of this study are the high quality of the trial conducted as well as the large sample size. In particular, the stepped-wedge design of this study - which enabled participants to act as their own controls - may allow for greater power in the analyses. However, this potential increase in power is offset by clustering at both the intra-individual and group level that decreases power. Also, whilst the stepped-wedge design improved feasibility for practical, ethical and financial reasons, it significantly added to the complexity of the analyses. A potential limitation of this study is that the tools used to measure the potential mediators may not have been optimal. For example, we used single items for fear avoidance rather than a complete fear-avoidance questionnaire. However, as the items have been used previously and the intervention delivered had a significant effect on the items as hypothesised we do not believe this affected our outcomes. The use of a self-reported measure of muscle strength may also be a cause for concern, however, the question has been validated previously (Strøyer et al. 2007) which gives us confidence in our results. Finally, our analysis makes assumptions as to the non-interdependence of the potential mediators investigated. While it is certainly possible that the variables are related, these interrelations need to be tested in future studies where multiple measures of each variable are included.

\section{Comparisons with other studies}

Although several studies have shown significant mediating effects of psychological factors such as catastrophizing (Spinhoven et al. 2004; Smeets et al. 2006; Hall et al. 2016), fear avoidance (Mansell et al. 2016a; Fordham et al. 2017; Whittle et al. 2017), pain self-efficacy (Fordham et al. 
2017) and pain related distress (Mansell et al. 2016b) on LBP outcomes, only one previous study has investigated physical factors, showing that frequency and engagement with every day activities mediated the effect of a CBT programme on disability (Fordham et al. 2017). As physical factors such as muscle strength (Taylor et al. 2014), physical activity (Heneweer et al. 2011) and sleep (Kelly et al. 2011; Alsaadi et al. 2014) have been linked to the development/prognosis of LBP we are unsure as to why physical factors have been largely ignored. Also, as previous studies have been limited to clinical populations, predominantly chronic LBP (Spinhoven et al. 2004; Smeets et al. 2006; Hall et al. 2016; Mansell et al. 2016a), when ours was conducted within a working population it seems probable that the pertinent mediating factors may change. For example, those without LBP are likely to have lower fearavoidance beliefs than those with pain (Basler et al. 2008) and therefore any further decrease may have no effect on LBP outcomes.

\section{Meaning of the study: explanations and implications}

The intervention conducted was effective in changing some of the potential mediators investigated (fear-avoidance beliefs and use of assistive devices) as well as improving clinical outcomes (Rasmussen et al. 2015). However, there was no significant relationship between these proposed mediators and LBP outcomes. This suggests that, whilst the intervention is working as planned, these potential mediators are not the mechanism through which the intervention acts, at least in our population. It may also be that the relationships these potential mediators have with LBP outcomes are more complex than that which we have proposed. For example, it may be that changing fear-avoidance beliefs do not help for LBP unless changing fear-avoidance also leads to changes in lifestyle physical activity. Despite the implied relationship between fear-avoidance beliefs and physical activity, this relationship cannot be assumed (Leeuw et al. 2007) and as physical activity was not measured in our study we cannot be sure whether this occurred.

Page 16 of 30 
It may also be that the heterogeneous nature of our population limited our ability to identify any significant indirect effects. Although we did conduct several sensitivity analyses their results were often unclear. For example, one of our measures of fear-avoidance increased the number of days with bothersome LBP in one model. However, this finding is inconsistent with what was hypothesised, does not agree with the previous literature (Mansell et al. 2016a; Fordham et al. 2017; Whittle et al. 2017) and thus could be a false positive. Other clinical features, such as a participant's LBP trajectory, may be more appropriate for subgroup analyses.

Our primary analysis shows that reducing perceived physical exertion leads to reductions in LBP intensity. Our sensitivity analysis suggests that this effect only holds true in those with pain at baseline. Thus, interventions which target reduction of physical exertion are likely to improve LBP outcomes, particularly in those who already have LBP. This intervention is already commonly recommended in the workplace, with workers placed on 'light duties' to enable them to continue working whilst they recover from their episode of LBP (Nguyen and Randolph 2007; Fenner 2013).

\section{Unanswered questions and future research}

A coherent model for LBP that adequately describes its complexity is needed for directing future research on the causes and mechanisms associated with LBP (including the effect of treatments). Also, further research is needed that investigates potential mediators not examined here (e.g. physical activity, sleep), as well as investigating these mediators in different populations. Ideally, all future clinical trials investigating interventions for LBP will also collect outcomes which allow for examination of the proposed mechanism. Potential mediators to be investigated should include both psychological and physical factors.

Page 17 of 30 


\section{Conclusions}

A multifaceted intervention consisting of participatory ergonomics, physical training, and CBT was able to change fear avoidance beliefs and use of assistive devices in the workplace. However, these changes did not explain the effect of any of the intervention components on days with LBP, LBP intensity and days with bothersome LBP. Interestingly, decreased perceived physical exertion at work (whilst not being affected by the intervention) did show significant reductions in pain intensity. Further research into other potential mediators, and in other populations, is required.

\section{Acknowledgements}

This study was funded by The Danish Working Environment Research Fund (Journal number: 201100813) and the National Prevention Fund (Journal number: 11-2-1a-113). MS is supported by a scholarship provided by the Chiropractic \& Osteopathic College of Australasia (COCA) Research Limited. GM is supported by a National Institute for Health Research (NIHR) School for Primary Care Research Seedcorn funding.

\section{Conflict of Interest}

The authors declare they have no conflicts of interest.

\section{References}

Alsaadi SM, McAuley JH, Hush JM, et al (2014) Poor sleep quality is strongly associated with subsequent pain intensity in patients with acute low back pain. Arthritis Rheumatol (Hoboken, NJ) 66:1388-94. doi: 10.1002/art.38329

Page 18 of 30 
Basler H-D, Luckmann J, Wolf U, Quint S (2008) Fear-avoidance beliefs, physical activity, and disability in elderly individuals with chronic low back pain and healthy controls. Clin J Pain 24:604-610

Borg E, Kaijser L (2006) A comparison between three rating scales for perceived exertion and two different work tests. Scand J Med Sci Sport 16:57-69. doi: 10.1111/j.16000838.2005.00448.x

Borg GA V (1962) Physical Performance and Perceived Exertion. Gleerup, Lund 1-32

d'Errico A, Viotti S, Baratti A, et al (2013) Low back pain and associated presenteeism among hospital nursing staff. J Occup Health 55:276-283. doi: 10.1539/joh.12-0261-OA

Dionne CE, Dunn KM, Croft PR, et al (2008) A consensus approach toward the standardization of back pain definitions for use in prevalence studies. Spine (Phila Pa 1976) 33:95-103. doi: 10.1097/BRS.0b013e31815e7f94

Driessen MT, Proper KI, van Tulder MW, et al (2010) The effectiveness of physical and organisational ergonomic interventions on low back pain and neck pain: a systematic review. Occup Environ Med 67:277-285. doi: 10.1136/oem.2009.047548

Fenner P (2013) Returning to work after an injury. Aust Fam Physician 42:182-185

Fordham B, Ji C, Hansen Z, et al (2017) Explaining How Cognitive Behavioural Approaches Work for Low Back Pain. Spine (Phila Pa 1976) 1. doi: 10.1097/BRS.0000000000002066

Hall AM, Kamper SJ, Emsley R, Maher CG (2016) Does pain-catastrophising mediate the effect of tai chi on treatment outcomes for people with low back pain? Complement Ther Med 25:61-66. doi: 10.1016/j.ctim.2015.12.013

Heneweer H, Staes F, Aufdemkampe G, et al (2011) Physical activity and low back pain: a systematic review of recent literature. Eur Spine J 20:826-45. doi: 10.1007/s00586-010$1680-7$

Hoy D, Bain C, Williams G, et al (2012) A systematic review of the global prevalence of low back pain. Arthritis Rheum 64:2028-2037. doi: 10.1002/art.34347

Page 19 of 30 
Jørgensen MB, Ektor-Andersen J, Sjøgaard G, et al (2011) A randomised controlled trial among cleaners-Effects on strength, balance and kinesiophobia. BMC Public Health 11:1-10. doi: $10.1186 / 1471-2458-11-776$

Kamper SJ, Apeldoorn AT, Chiarotto A, et al (2015) Multidisciplinary biopsychosocial rehabilitation for chronic low back pain: Cochrane systematic review and meta-analysis. BMJ 350:h444. doi: 10.1136/bmj.h444

Kelly GA, Blake C, Power CK, et al (2011) The association between chronic low back pain and sleep: a systematic review. Clin J Pain 27:169-81. doi: 10.1097/AJP.0b013e3181f3bdd5

Laisné F, Lecomte C, Corbière M (2012) Biopsychosocial predictors of prognosis in musculoskeletal disorders: a systematic review of the literature (corrected and republished)*. Disabil Rehabil 34:1912-1941. doi: 10.3109/09638288.2012.729362

Lee H, Hübscher M, Moseley GL, et al (2015) How does pain lead to disability? A systematic review and meta-analysis of mediation studies in people with back and neck pain. Pain 156:988-97. doi: 10.1097/j.pain.0000000000000146

Lee H, Mansell G, McAuley JH, et al (2017) Causal mechanisms in the clinical course and treatment of back pain. Best Pract Res Clin Rheumatol. doi: 10.1016/j.berh.2017.04.001

Lee H, McAuley JH, Hübscher M, et al (2016) Does changing pain-related knowledge reduce pain and improve function through changes in catastrophizing? Pain 157:1. doi: 10.1097/j.pain.0000000000000472

Leeuw M, Goossens MEJB, Linton SJ, et al (2007) The fear-avoidance model of musculoskeletal pain: current state of scientific evidence. J Behav Med 30:77-94. doi: 10.1007/s10865-006$9085-0$

Linton SJ, Boersma K, Jansson M, et al (2005) The effects of cognitive-behavioral and physical therapy preventive interventions on pain-related sick leave: a randomized controlled trial. Clin J Pain 21:109-119. doi: 10.1097/00002508-200503000-00001

Linton SJ, Nicholas M, Macdonald S (2010) Development of a Short Form of the Örebro Page 20 of 30 
Mechanisms for Reducing Low Back Pain

Musculoskeletal Pain Screening Questionnaire. Spine (Phila Pa 1976) 36:1891-1895. doi:

10.1097/BRS.0b013e3181f8f775

Mannion AF, Horisberger B, Eisenring C, et al (2009) The Association Between Beliefs About

Low Back Pain and Work Presenteeism. J Occup Environ Med 15-18. doi:

10.1097/JOM.0b013e3181beac69

Mansell G, Hill JC, Main C, et al (2016a) Mediators of Treatment Effect in the Back In Action Trial: Using latent growth modelling to take change over time into account

Mansell G, Hill JC, Main C, et al (2016b) Exploring What Factors Mediate Treatment Effect: Example of the STarT Back Study High-Risk Intervention. J Pain 17:1237-1245. doi: 10.1016/j.jpain.2016.08.005

Mansell G, Kamper SJ, Kent P (2013) Why and how back pain interventions work: What can we do to find out? Best Pract Res Clin Rheumatol 27:685-697. doi: 10.1016/j.berh.2013.10.001

Murray CJL, Lopez AD (2013) Measuring the global burden of disease. N Engl J Med 369:44857. doi: 10.1056/NEJMra1201534

Nguyen TH, Randolph DC (2007) Nonspecific low back pain and return to work. Am Fam Physician 76:

Rabe-Hesketh S, Skrondal A, Pickles A (2005) Maximum likelihood estimation of limited and discrete dependent variable models with nested random effects. J Econom 128:301-323. doi: 10.1016/j.jeconom.2004.08.017

Rasmussen CDN, Holtermann A, Bay H, et al (2015) A multi-faceted workplace intervention for low back pain in nurses' aides: a pragmatic stepped wedge cluster randomised controlled trial. Pain 156:1786-1794. doi: 10.1097/j.pain.0000000000000234

Rasmussen CDN, Holtermann A, Mortensen OS, et al (2013) Prevention of low back pain and its consequences among nurses' aides in elderly care: a stepped-wedge multi-faceted clusterrandomized controlled trial. BMC Public Health 13:1088. doi: 10.1186/1471-2458-13-1088

Rasmussen CDN, Lindberg NK, Ravn MH, et al (2017) Processes, barriers and facilitators to Page 21 of 30 
implementation of a participatory ergonomics program among eldercare workers. Appl Ergon 58:491-499. doi: 10.1016/j.apergo.2016.08.009

Rivilis I, Van Eerd D, Cullen K, et al (2008) Effectiveness of participatory ergonomic interventions on health outcomes: A systematic review. Appl Ergon 39:342-358. doi: 10.1016/j.apergo.2007.08.006

Schofield DJ, Callander EJ, Shrestha RN, et al (2015) Back problems, comorbidities, and their association with wealth. Spine J 15:34-41. doi: 10.1016/j.spinee.2014.06.018

Schofield DJ, Shrestha RN, Passey ME, et al (2008) Chronic disease and labour force participation among older Australians. Med J Aust 189:447-450

Schofield DJ, Shrestha RN, Percival R, et al (2011) Early retirement and the financial assets of individuals with back problems. Eur Spine J 20:731-736. doi: 10.1007/s00586-010-1647-8

Sjøgaard G, Justesen JB, Murray M, et al (2014) A conceptual model for worksite intelligent physical exercise training--IPET--intervention for decreasing life style health risk indicators among employees: a randomized controlled trial. BMC Public Health 14:652. doi: $10.1186 / 1471-2458-14-652$

Smeets RJEM, Vlaeyen JWS, Kester ADM, Knottnerus JA (2006) Reduction of Pain Catastrophizing Mediates the Outcome of Both Physical and Cognitive-Behavioral Treatment in Chronic Low Back Pain. J Pain 7:261-271. doi: 10.1016/j.jpain.2005.10.011

Spinhoven P, Ter Kuile M, Kole-Snijders AMJ, et al (2004) Catastrophizing and internal pain control as mediators of outcome in the multidisciplinary treatment of chronic low back pain. Eur J Pain 8:211-219. doi: 10.1016/j.ejpain.2003.08.003

Strøyer J, Jensen LD, Avlund K, et al (2007) Validity and reliability of self-assessed physical fitness using visual analogue scales. Percept Mot Ski 104:519-533. doi: 10.2466/PMS.104.2.519-533

Taylor JB, Goode AP, George SZ, Cook CE (2014) Incidence and risk factors for first-time incident low back pain: A systematic review and meta-analysis. Spine J 14:2299-2319. doi: 
10.1016/j.spinee.2014.01.026

Tveito TH, Hysing M, Eriksen HR (2004) Low back pain interventions at the workplace: A systematic literature review. Occup Med (Chic Ill) 54:3-13. doi: 10.1093/occmed/kqg109 van Tulder MW, Ostelo R, Vlaeyen JW, et al (2001) Behavioral treatment for chronic low back pain: a systematic review within the framework of the Cochrane Back Review Group. Spine (Phila Pa 1976) 26:270-281. doi: 10.1097/00007632-200102010-00012

Vos T, Flaxman AD, Naghavi M, et al (2012) Years lived with disability (YLDs) for 1160 sequelae of 289 diseases and injuries 1990-2010: a systematic analysis for the Global Burden of Disease Study 2010. Lancet 380:2163-96. doi: 10.1016/S0140-6736(12)61729-2 Whittle R, Mansell G, Jellema P, van der Windt D (2017) Applying causal mediation methods to clinical trial data: What can we learn about why our interventions (don't) work? Eur J Pain (United Kingdom) 21:614-622. doi: 10.1002/ejp.964

Wieser S, Horisberger B, Schmidhauser S, et al (2011) Cost of low back pain in Switzerland in 2005. Eur J Heal Econ 12:455-467. doi: 10.1007/s10198-010-0258-y 
Figure 1. Overview of the stepped-wedge design used for the randomised-controlled trial of a multifaceted intervention in workers in elderly care.

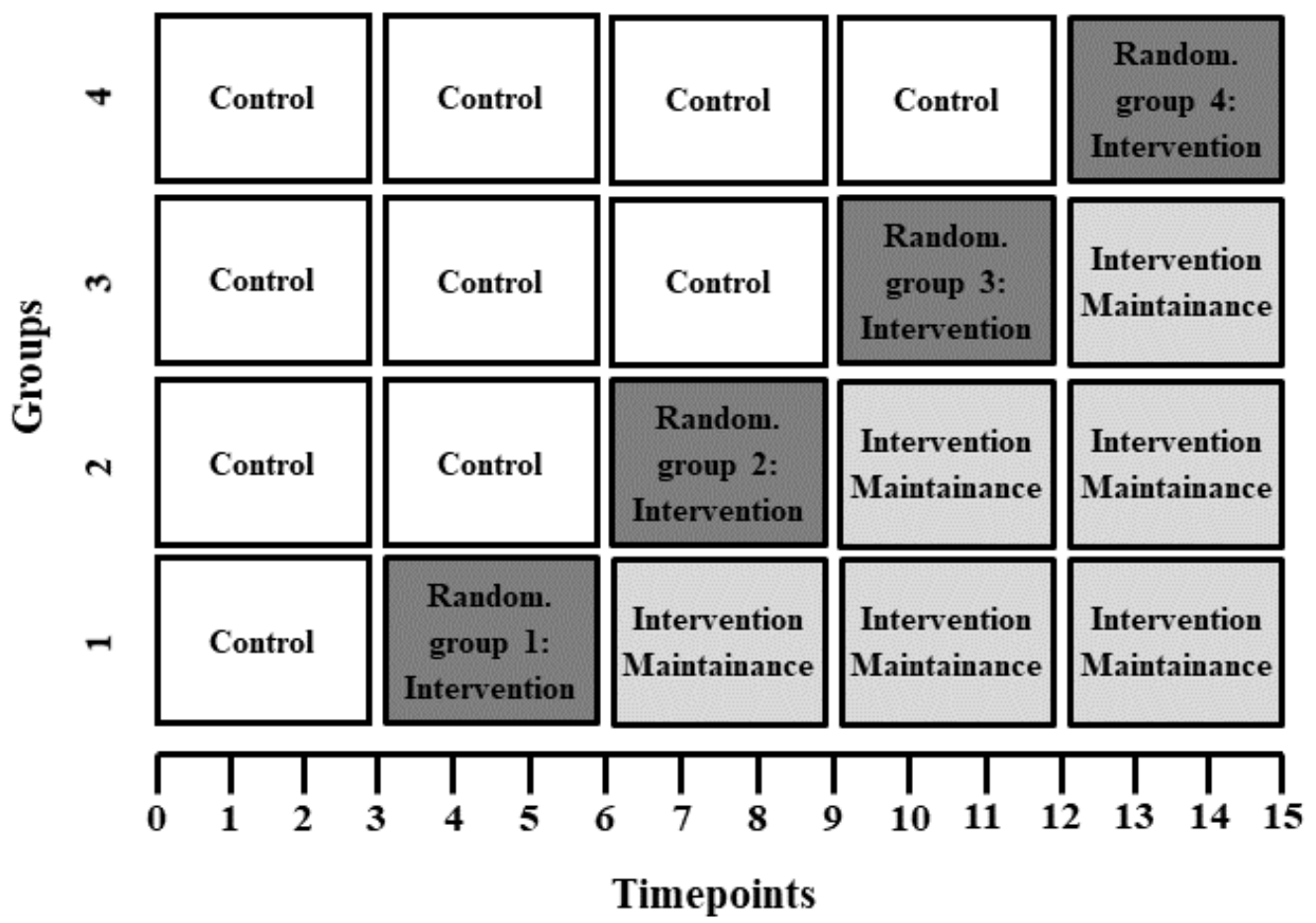

Page 24 of 30 
Figure 2. Timeline for each component of the multifaceted intervention for reducing low back pain in workers in elderly care.

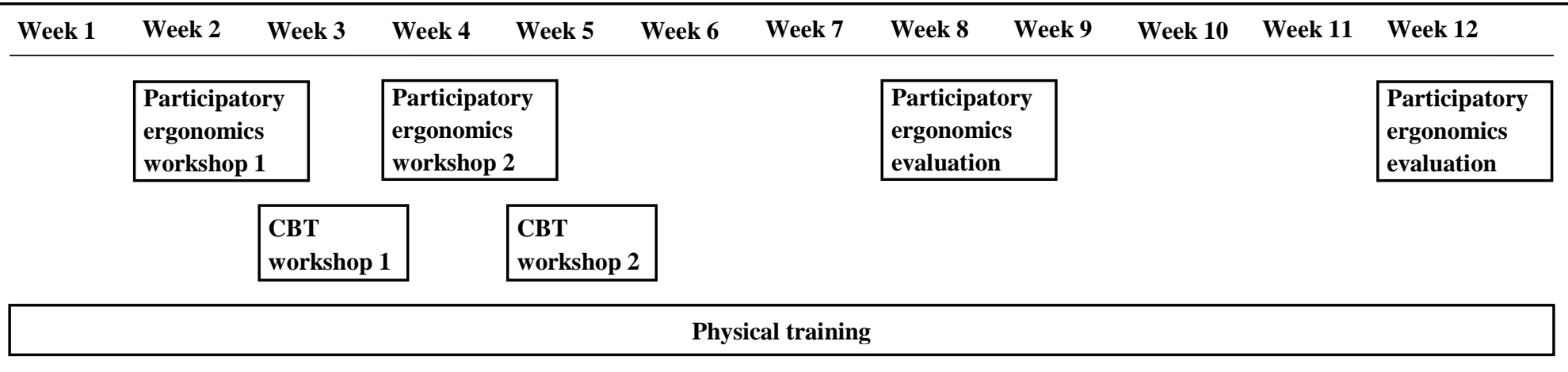


Figure 3. Hypothesised pathways of action for the effect of the multifaceted intervention on low back pain outcomes.

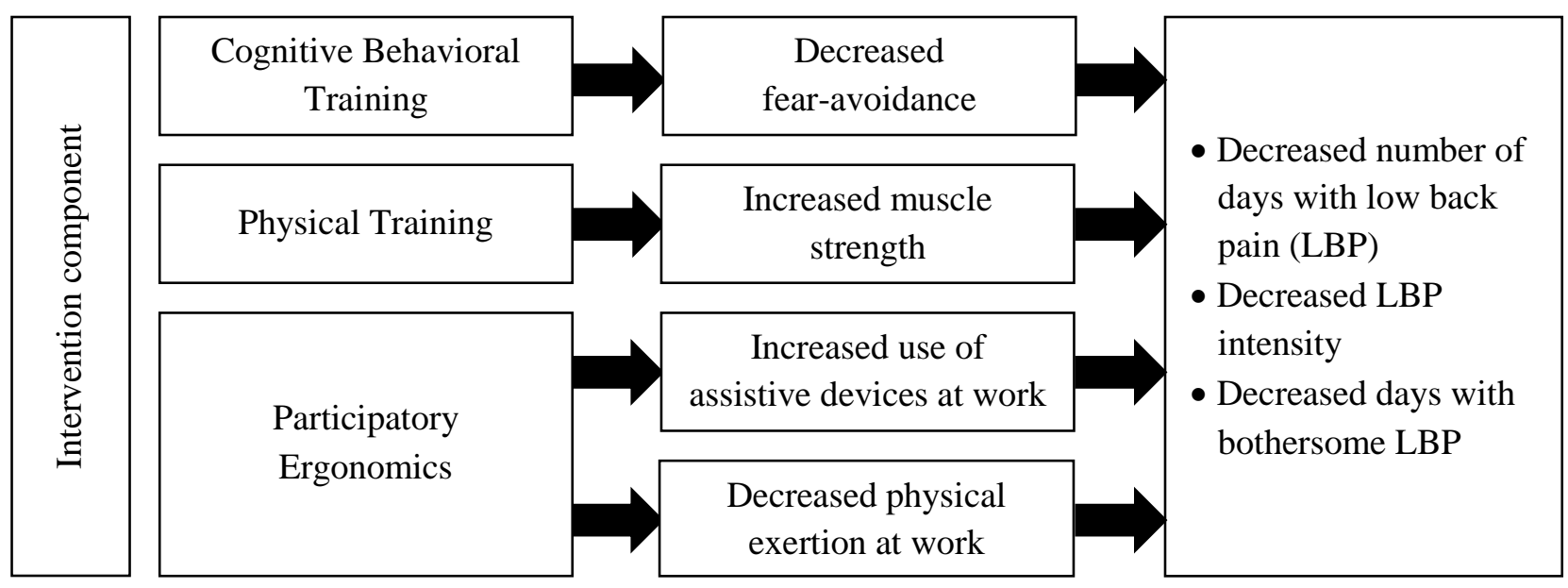


Table 1. Demographics and baseline characteristics for workers in elderly care participating in the mediation analyses

\begin{tabular}{|c|c|}
\hline Demographics / baseline characteristics & n $(\%)$ or mean $(\mathrm{SD})$ \\
\hline Gender, female $(n=587)^{a}$ & $549(93.5 \%)$ \\
\hline \multicolumn{2}{|l|}{ Length of time in occupation $(\mathrm{n}=530)$} \\
\hline One year or less & $77(14.5 \%)$ \\
\hline Two to 10 years & $226(42.6 \%)$ \\
\hline Greater than 10 years & $227(42.8 \%)$ \\
\hline \multicolumn{2}{|l|}{ Work shift $(n=568)$} \\
\hline Day & $456(80.3 \%)$ \\
\hline Afternoon / evening & $112(19.7 \%)$ \\
\hline \multicolumn{2}{|l|}{ Place of work $(n=522)$} \\
\hline Nursing home & $233(44.6 \%)$ \\
\hline Homecare & $289(55.4 \%)$ \\
\hline \multicolumn{2}{|l|}{ Type of work $(n=587)$} \\
\hline Nurses' aides & $526(89.6 \%)$ \\
\hline Service workers & $61(10.4 \%)$ \\
\hline \multicolumn{2}{|l|}{ Education $(n=573)$} \\
\hline Unskilled & $35(6.1 \%)$ \\
\hline Low skilled ( $<2$ years of tertiary education) & $373(65.1 \%)$ \\
\hline High skilled ( $\geq 2$ years of tertiary education) & $165(28.8 \%)$ \\
\hline Born in Denmark $(n=575)$ & $510(88.7 \%)$ \\
\hline Current smoker $(n=575)$ & $163(28.4 \%)$ \\
\hline BMI $(n=536)$ & $34.4(8.4)$ \\
\hline \multicolumn{2}{|l|}{ Self-rated health $(\mathrm{n}=575)$} \\
\hline Very good & $20(3.5 \%)$ \\
\hline Good & $170(29.6 \%)$ \\
\hline Fair & $317(55.1 \%)$ \\
\hline Poor & $66(11.5 \%)$ \\
\hline Very poor & $2(0.4 \%)$ \\
\hline \multicolumn{2}{|l|}{ LBP in the past four weeks $(n=587)$} \\
\hline At least one day with LBP & $353(60.1 \%)$ \\
\hline Number of days with LBP & $5.5(7.9)$ \\
\hline Maximal pain intensity & $2.9(3.0)$ \\
\hline Number of days with bothersome LBP & $2.9(5.6)$ \\
\hline \multicolumn{2}{|c|}{$\begin{array}{l}\text { BMI - body mass index; LBP - low back pain. } \\
\text { a As participants could contribute to both intervention and control groups the number analysec } \\
\text { is higher than the number participating. }\end{array}$} \\
\hline
\end{tabular}


Figure 4. Effect of the potential mediators on the number of days with low back pain

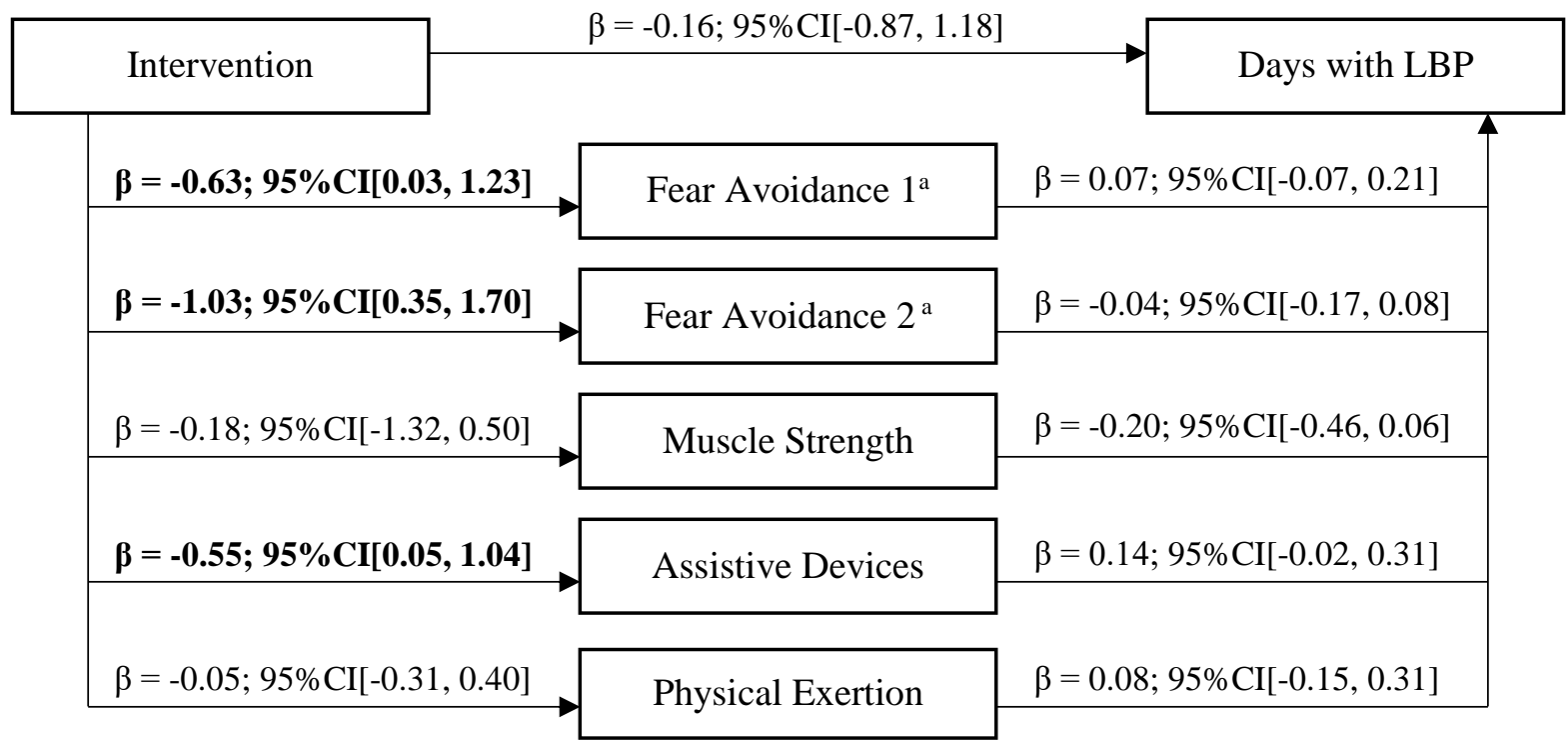

${ }^{\text {a }}$ Fear-avoidance was measured using two items from the Örebro Musculoskeletal Pain Screening Questionnaire 
Figure 5. Effect of the potential mediators on low back pain intensity

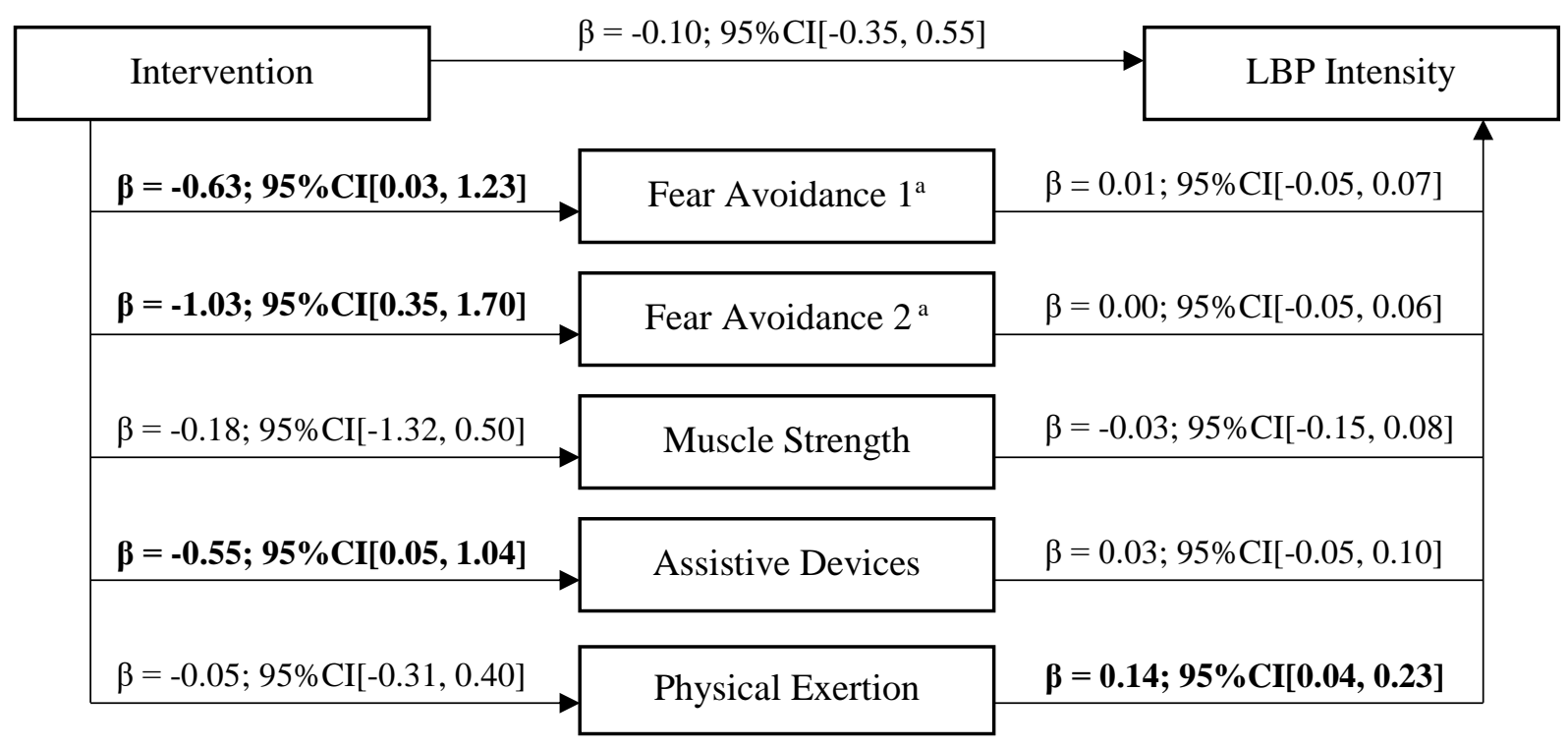

${ }^{\text {a }}$ Fear-avoidance was measured using two items from the Örebro Musculoskeletal Pain Screening Questionnaire 
Figure 6. Effect of the potential mediators on the number of days with bothersome low back pain

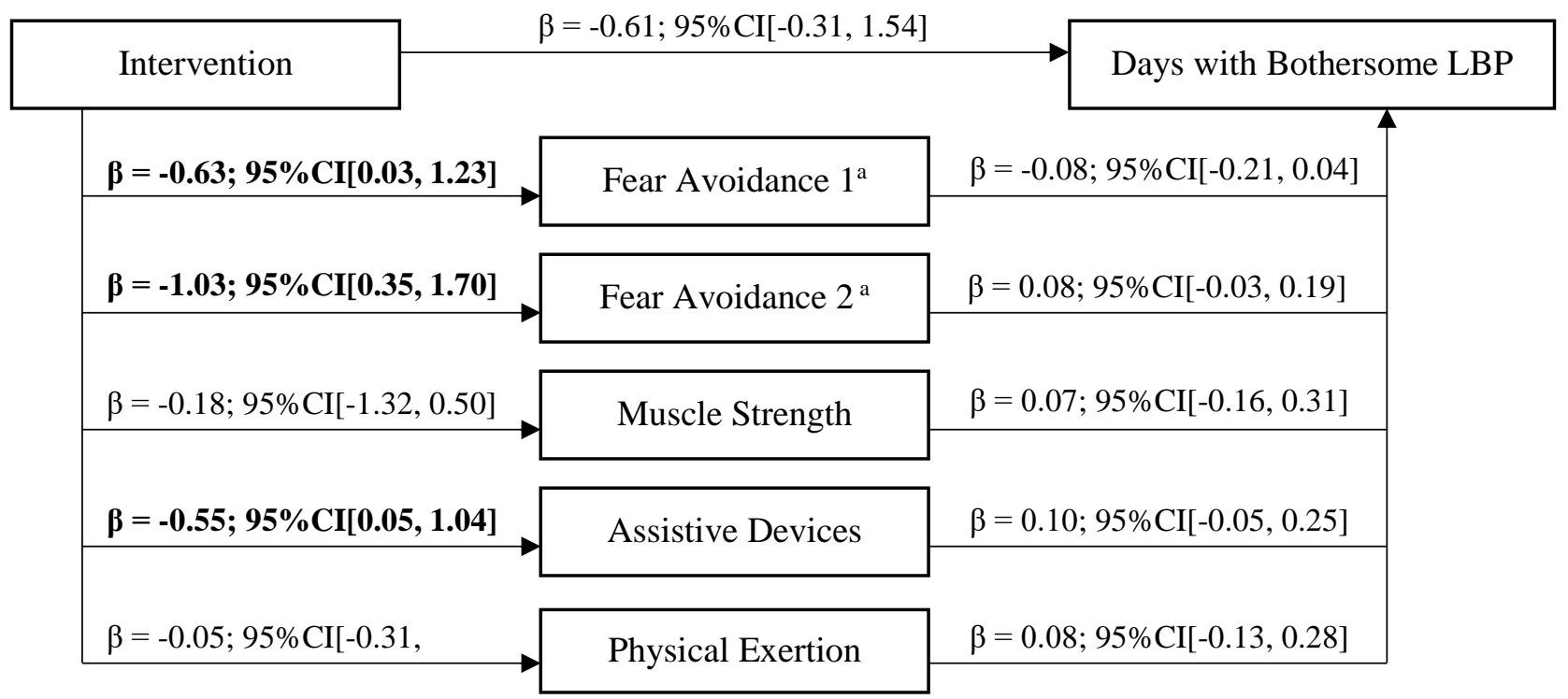

${ }^{a}$ Fear-avoidance was measured using two items from the Örebro Musculoskeletal Pain Screening Questionnaire 